\title{
THREE-DIMENSIONAL ELASTIC CRACK TIP INTERACTIONS WITH TRANSFORMATION STRAINS AND DISLOCATIONS
}

\author{
James R. Rice \\ Division of Applied Sciences, Harvard University, Cambridge, MA 02138. U.S.A.
}

\begin{abstract}
Three-dimensional elastic intcractions between a half-plane crack and sources of internal stress such as transformation strains and dislocations are analyzed. These interactions include the stress intensity factors induced along the crack front by the source and the overall energy change and stress field induced in the source region owing to the presence of the crack. The analysis is based on the author's recent extension of "weight function" methods of threedimensional crack analysis[1] and principal results of that extension are summarized at the outset here.
\end{abstract}

\section{INTRODUCTION}

In this study, recent developments[1] in three-dimensional elastic crack analysis are applied to deriving the interaction between a source of internal stress and a crack tip. The source is represented as some region that is given an Eshelby transformation of its stress-free state. This representation includes, as a limiting case, an arbitrary dislocation on a planar cut. As particular cases for which the mathematics is relatively tractable, explicit results are given for the tensile stress intensity factor due to arbitrary dilatant transformations in an isotropic material, and for the intensity factor and stress field associated with an opening dislocation (e.g. a prismatic dislocation loop) on a cut that is coplanar with the crack.

Understanding the interaction between an arbitrary source and the crack referred to above involves calculating the stress intensity factors induced along the crack tip by the source, and also calculating the energy and image like contributions to stresses or energetic forces (i.e. configurational forces) exerted on the source owing to the presence of the crack. The recent developments[1] which facilitate such calculations are the analysis of first-order variations in three-dimensional elastic fields associated with variations in location of the tip of a planar crack and the use of such analyses to extend and apply "weight function" $[2,3,4]$ concepts for crack analysis in the threedimensional regime.

The present work is dedicated to the memory of Alicia Golebiewska Herrmann, whose interests included the theory of dislocations, cracks, and other defects in solids and of the energetic forces which act upon them.

\section{THREE-DIMENSIONAL WEIGHT FUNCTIONS FOR CRACKS}

Consider a half-plane crack in an infinite elastic body. The crack lies on the plane $y=0$ and its tip is parallel to the $z$ axis along $x=a$ such that the region $x<a$ is cracked. As is well known, in such circumstances arbitrary loadings induce a singularity at the crack tip such that stress components $\sigma_{i j}(i, j=x, y, z)$ ahead of the tip on $y$ $=0$ vary like

$$
\left.\left(\sigma_{y y}, \sigma_{y x}, \sigma_{y z}\right)\right|_{y=0} \sim\left(K_{1}, K_{2}, K_{3}\right) / \sqrt{2 \pi(x-a)}
$$

as $x \rightarrow a$. The $K$ 's are stress intensity factors and may vary with position $z$ along the crack front. They appear also in the expression

$$
G=\Lambda_{\alpha \beta} K_{\alpha} K_{\beta}
$$


(Greek indices have range 1, 2, 3 and the summation convention is followed with repeated indices) for the Irwin energy release per unit area of crack advance. The coefficients $\Lambda_{\alpha \beta}$ are symmetric, $\Lambda_{\beta \alpha}=\Lambda_{\alpha \beta \beta}$, and for an isotropic material $\Lambda_{\alpha \beta 3}$ is diagonal with

$$
\Lambda_{11}=\Lambda_{22}=(1-v) / 2 \mu, \quad \Lambda_{33}=1 / 2 \mu
$$

( $\mu=$ shear modulus, $\nu=$ Poisson ratio). Following Stroh[5] and Barnett and Asarol6], for anisotropic solids $\Lambda_{\alpha \beta}$ can be expressed as a numerical factor times the inverse of a matrix appearing (pre- and post-multiplied by Burgers vector) in the pre-logarithmic energy factor for a straight dislocation line lying parallel to the direction of the crack tip. The displacement discontinuity across the crack surfaces very near the crack tip may be expressed in terms of the $K^{\prime} s$ and $\Lambda^{\prime}$ 's by

$$
\left(\Delta u_{y}, \Delta u_{x}, \Delta u_{z}\right) \sim\left(\Lambda_{1 \beta} K_{\beta}, \Lambda_{2 \beta} K_{\beta}, \Lambda_{3 \beta} K_{\beta}\right) 8 \sqrt{(a-x) / 2 \pi}
$$

where $\Delta u_{i}=u_{i}\left(x, 0^{+}, z\right)-u_{i}\left(x, 0^{-}, z\right)$. Thus the terms $\Lambda_{\alpha \beta} K_{\beta}, \alpha=1,2,3$, may be regarded as displacement intensity factors.

In [1], three vector functions $\underline{h}_{\alpha}=\underline{h}_{\alpha}\left(r, z^{\prime}, a\right)$ of position $\underline{r}(=(x, y, z))$ in the body were introduced. Here $\alpha=1,2,3$. These functions are also associated with a location $z=z^{\prime}$ along the crack front and they depend, of course, also on $a$, which measures the position of the crack front. The functions are called "weight functions" and they are universal for a given geometry of cracked body in the sense of having no dependence on the particular distribution of loading forces to which the body is subjected. For the half-plane crack in an infinite homogeneous body, translational invariance requires that the $h_{\alpha}\left(r, z^{\prime}, a\right)$ be dependent only on $x-a, y$, and $z-z^{\prime}$.

The weight functions have the following two properties: The stress intensity factors induced at the location $z=z^{\prime}$ along the crack tip by an arbitrary distribution of body force $\underline{f}=\underline{f}(\underline{r})$ per unit volume are given by

$$
K_{\alpha}\left(z^{\prime}\right)=\int_{V} \underline{h}_{\alpha}\left(\underline{r}, z^{\prime}, a\right) \cdot \underline{f}(\underline{r}) \mathrm{d} V(\underline{r})
$$

where $d V(r)$ denotes an element of volume and the integral extends over all loaded elements. Allso, if the crack tip is moved from $x=a$ to the neighboring position $x=$ $a+\delta a(z)$, where $\delta a(z)$ is an arbitrary function of position $z$ along the tip, while the body is subject to some system of loadings inducing stress intensity factors $K_{\beta}(z)$ along the initial straight crack tip, the associated variation in displacement field $\underline{u}=\underline{u}(r)$ is

$$
\delta \underline{u}(r)=2 \int_{-\infty}^{+\infty} \Lambda_{\mathrm{\alpha} \beta} \underline{h}_{\alpha}\left(\underline{r}, z^{\prime}, a\right) K_{\beta}\left(z^{\prime}\right) \delta a\left(z^{\prime}\right) \mathrm{d} z^{\prime}
$$

to first order in $\delta a\left(z^{\prime}\right)$. Thus the displacement intensity factors $\Lambda_{\alpha \beta} K_{\beta}$ times $\delta a$ are the source terms, weighted with the $\underline{h}_{\alpha}$, to form $\delta \underline{u}$. Later it will be seen why the second property necessarily follows from the first; see [1] also.

The functions $h_{\alpha}$ are expressed in [1] for isotropic homogeneous materials with half-plane cracks in terms of certain rather formidable double integrals. An explicit solution was also developed for $\underline{h}_{1}$ (denoted as $\underline{h}$ in the relevant part of [1]) by considering a crack under arbitrary tensile (mode 1) loading, and by directly formulating and solving the three-dimensional elasticity problem for the first order variation $\delta u(r)$ associated with arbitrary $\delta a(z)$. Probably a similar approach based, e.g., on the representation of shear mode solutions for the half-plane crack as given by Meade and Keer[7], will prove a convenient route to explicit forms for $\underline{h}_{2}$ and $\underline{h}_{3}$. The results for the $x, y, z$ components of $\underline{h}_{1}$ are 


$$
\begin{aligned}
& h_{1 . x}=-[1 / 2(1-v)] \partial(L+y H) / \partial x \\
& h_{1 .}=H-[1 / 2(1-v)] y \partial H / \partial y \\
& h_{1:}=-[1 / 2(1-v)] \partial(L+y H) / \partial z,
\end{aligned}
$$

where $H$ and $L$ are harmonic in $x, y, z$ and given by

$$
H\left(x-a, y, z-z^{\prime}\right)=\frac{\left(1 / 2 \pi^{3}\right)^{1 / 2} \operatorname{Im}\left[(x-a+i y)^{1 / 2}\right]}{(x-a)^{2}+y^{2}+\left(z-z^{\prime}\right)^{2}},
$$

with branch cut on the crack surface and

$$
L\left(x-a, y, z-z^{\prime}\right)=-(1-2 v) \int_{y}^{x} H\left(x-a, \bar{y}, z-z^{\prime}\right) \mathrm{d} \bar{y} .
$$

It may be noted also, as required for subsequent applications, that

$$
\begin{aligned}
& h_{1 y, y} \equiv \partial h_{1, y} / \partial y=[(1-2 v) / 2(1-v)] \partial H / \partial y-[1 / 2(1-v)] y \partial^{2} H / \partial y^{2}, \\
& h_{1, j, j} \equiv h_{1, r . . x}+h_{1, . . y}+h_{1, .:}=[(1-2 v) /(1-v)] \partial H / \partial y .
\end{aligned}
$$

Here Latin indices such as $j$ (but excepting $x, y$, and $z$ ) range over the values $x, y, z$ with summation on repeated indices; the comma denotes partial differentiation.

The following result was also derived in [I] for a planar crack with a slightly nonstraight front in an isotropic homogeneous solid under general loadings that cause mode 1 tension at the tip. For present purposes, let $K_{i}^{0}[z, a]$ denote the tensile stress intensity which the given loadings induce at location $z$ along the tip when the tip is straight and is located at $x=a$. For example, $K_{i}^{0}\left[z^{\prime}, a\right]$ is given by the right side of eqn (5) with $\alpha=1$. Then for the case when the tip deviates slightly from straightness, lying along the curve $x=b(z)$ in the plane $y=0$, one has

$$
K_{1}(z)=K_{1}^{0}[z, b(z)]+\frac{1}{2 \pi} \int_{-\infty}^{+\infty} \frac{K_{1}^{0}\left[z^{\prime}, b(z)\right]\left[b\left(z^{\prime}\right)-b(z)\right]}{\left(z^{\prime}-z\right)^{2}} \mathrm{~d} z^{\prime}
$$

to first order in $b\left(z^{\prime}\right)-b(z)$ for the mode 1 intensity at $z$; the integral is interpreted in a principal value sense. This expression improves upon a result of Meade and $\operatorname{Keer[7]}$ as discussed in [1].

Knowledge of the weight functions $h_{\alpha}$ lets one express the displacement field everywhere in the half-plane cracked body provided that the field is known when the given loadings act but no crack is present. To see why, let $\delta a$ be uniform in $z^{\prime}$ in eqn (6) and divide by $\delta a$. This gives

$$
\left.\frac{\partial \underline{u}}{\partial a}=2 \int_{-\infty}^{+\infty} \Lambda_{\alpha \beta} \underline{h_{\alpha}}\left(\underline{r}, z^{\prime}, a\right) \int_{V} \underline{h_{\beta}}\left(\underline{\tilde{r}}, z^{\prime}, a\right) \cdot \underline{f} \underline{\underline{r}}\right) \mathrm{d} V(\underline{\tilde{r}}) \mathrm{d} z^{\prime},
$$

where eqn (5) for $K_{\beta}$ has been used. Suppose that the dyad $G^{\text {Kelv }}(r-\vec{r})$, with components $G_{i j}^{\text {Kelv }}$, is the Kelvin Green's function for the infinite uncracked body, or is the generalization of Kelvin function for an infinite anisotropic uncracked body. Then, when there is no crack present (i.e. when $a=-x$ )

$$
\underline{u}(\underline{r})=\int_{V} \underline{G}^{\mathrm{Kelv}}(\underline{r}-\underline{\tilde{n}}) \underline{f}(\underline{\tilde{r}}) \mathrm{d} V(\underline{\tilde{r}}) .
$$

If we add to this the integral of $\partial u / \partial a$ from $-x$ to 0 , we get the displacement field for a body subjected to the given loadings and containing a half-plane crack on $y=0$ with tip along the $z$ axis: 


$$
\begin{aligned}
\underline{u}(\underline{r})= & \int_{V} \underline{G}^{\mathrm{Kelv}}(\underline{r}-\underline{\tilde{r}}) \cdot \underline{f} \underline{(\tilde{r})} \mathrm{d} V(\underline{\tilde{r}}) \\
& \left.+2 \int_{-\infty}^{0} \int_{-\infty}^{+\infty} \Lambda_{\mathrm{\alpha} \beta} \underline{h_{\alpha}}\left(\underline{r}, z^{\prime}, a\right) \int_{V} \underline{h_{\beta}}\left(\underline{\dot{r}}, z^{\prime}, a\right) \cdot \underline{f} \underline{\underline{r}}\right) \mathrm{d} V(\underline{\tilde{r}}) \mathrm{d} z^{\prime} \mathrm{d} a .
\end{aligned}
$$

If an isotropic solid is subjected to symmetric loadings $f$ which induce only mode 1 tension conditions along its tip, the volume integrals in eqns (12) and (14) vanish for $\beta=2$ and 3 . In such cases, eqn (14) reduces to

$$
\begin{aligned}
\underline{u}(\underline{r})= & \int_{V} \underline{G}^{\mathrm{Kelv}}(\underline{r}-\underline{\tilde{r}}) \cdot \underline{f}(\underline{\tilde{r}}) \mathrm{d} V(\underline{\tilde{r}}) \\
& +\frac{1-v}{\mu} \int_{-\infty}^{0} \int_{-\infty}^{+\infty} \underline{h}_{1}\left(\underline{r}, z^{\prime}, a\right) \int_{V} \underline{h}_{1}\left(\underline{\tilde{r}}, z^{\prime}, a\right) \cdot \underline{f}(\underline{\tilde{r}}) \mathrm{d} V(\underline{\tilde{r}}) \mathrm{d} z^{\prime} \mathrm{d} a .
\end{aligned}
$$

When we read-in the components of $h_{1}$ from eqns (7), and move the integration operation on $z^{\prime}$ above inside the differential or integral operations on other variables in eqns $(7,9)$, the integration on $z^{\prime}$ is found to be elementary in each case. For all components of $h_{1 i}\left(\underline{r}, z^{\prime}, a\right) \underline{h}_{1 j}\left(\bar{r}, z^{\prime}, a\right)$ we need the integral

$$
\begin{aligned}
\int_{-\infty}^{+x} \frac{\mathrm{d} z^{\prime}}{\left[(x-a)^{2}+y^{2}+\left(z-z^{\prime}\right)^{2}\right]\left[(\tilde{x}-a)^{2}+\bar{y}^{2}+\left(\tilde{z}-z^{\prime}\right)^{2}\right]} \\
=\pi(1 / \rho+1 / \bar{\rho}) /\left[(z-\bar{z})^{2}+(\rho+\bar{\rho})^{2}\right],
\end{aligned}
$$

established by residue methods, where

$$
\rho=\sqrt{(x-a)^{2}+y^{2}}, \quad \tilde{\rho}=\sqrt{(\tilde{x}-a)^{2}+\tilde{y}^{2}} .
$$

The integral on a in eqn (15) is more formidable but is carried out subsequently for a special application.

\section{STRESS INTENSITIES DUE TO TRANSFORMATIONS AND DISLOCATIONS}

Suppose that the cracked solid is given some distribution of transformation strain $\underline{\epsilon}^{T}=\underline{\epsilon}^{T}(\underline{r})$ throughout a region $V$. This is to be understood in the sense that

$$
\sigma_{i j}=C_{i j m n}\left(u_{m, n}-\epsilon_{m n}^{T}\right)
$$

where $\underline{C}$ is the same modulus tensor as before transformation and has the usual symmetries. By a well-known argument used, e.g. for analysis of transformation of an inclusion by Eshelby and for stress analysis in presence of plastic strain by Lin, and earlier by Duhamel for thermal stress analysis, the displacement field produced by the $\underline{\epsilon}^{T}$ distribution is the same as that produced in an identical body with $\epsilon^{T}=0$ subject to a certain effective force field. That is, the equilibrium equations read

$$
\sigma_{i j, i}+f_{j}=\left(C_{i j m n} u_{m, n}\right)_{, i}+f_{j}-\left(C_{i j m n} \epsilon_{i n n}^{T}\right)_{. i}=0
$$

and on traction-free elements of boundary, such as crack surfaces in the present case, having outer normal $\underline{n}$

$$
T_{j}=n_{i} \sigma_{i j}=n_{i} C_{i j m n} u_{m, n}-n_{i} C_{i j m n} \epsilon_{m n}^{T}=0 .
$$

Thus the displacement field induced by $\underline{\epsilon}^{T}$ is the same as that induced by the effective body. force distribution

$$
f_{j}^{\text {eff }}=-\left(C_{i j m n} \epsilon_{m n}^{T}\right)_{i}
$$


throughout the transformed region $V$ plus a Dirac singular layer of effective body force along the crack surfaces equivalent to tractions

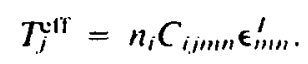

Inserting this effective force field into eqn (5) and applying the divergence theorem. which results in cancellation of the integral of $h_{\mathrm{c}, j} T_{j}^{\mathrm{tr}}$ along the traction-free surfaces, one sees that the distribution of transformation strain induces stress intensity factors

$$
K_{\mathrm{u}}\left(z^{\prime}\right)=\int_{V} h_{\mathrm{\alpha}, j, i}\left(\underline{r}, z^{\prime}, a\right) C_{i j m, n !} \epsilon_{m, n}^{\prime}(\underline{r}) \mathrm{d} V(\underline{r})
$$

at location $z^{\prime}$ along the crack front. Here the differentiation in $h_{\alpha x, i,}\left(r . z^{\prime}, a\right)$ is with respect to the $i$ th component of $r, i=x, y$, or $z$. The expression for $\bar{K}_{\mathrm{a}}$ is analogous to that derived on the basis of two-dimensional weight function theory by McMeeking and Evans [8] for isotropic cracked solids with uniformly transformed cylindrical regions with axis parallel to the tip of a plane-strain crack: the same two-dimensional problem had been solved by direct elasticity calculations by Hutchinson[9, 10]. Equation (23) reduces to those results when we consider the isotropic solid, make $\underline{\epsilon}^{\prime}$ independent of $z$, and integrate in $z$ over $-x$ to $+x$.

Dislocations on some cut surface $A$ can also be considered: let the sides of $A$ be denoted + and - let $N$ be the normal to $A$ pointing from - to + , and $\Delta u=u(+)$ - $\underline{u}(-)$ be the dislccation. Then to represent a dislocation $\underline{\epsilon}^{\gamma}$ is regarded as Dirac singular on $A$, and zero outside $A$, such that if we integrate $\epsilon_{m n}^{\prime}$ over some volume element $\delta V$ that includes area $\delta A$ of $A$ there results

$$
\int_{\delta V} \epsilon_{m m}^{\gamma} \mathrm{d} V=\frac{1}{2} \int_{\delta A}\left(N_{m} \Delta u_{n}+N_{n} \Delta u_{m}\right) \mathrm{d} A
$$

Thus, the resulting stress intensity factor distribution along the crack tip is

$$
K_{\mathrm{c}}\left(z^{\prime}\right)=\int_{A} h_{\alpha j . i}\left(\underline{r}, z^{\prime}, a\right) C_{i j m m} N_{n}(\underline{r}) \Delta u_{m}(\underline{r}) \mathrm{d} A(\underline{r})
$$

The full expression for the displacement field induced by the transformation strain is analogous to eqn (14) and its derivation again begins with eqn (6) which remains valid in this case. Thus

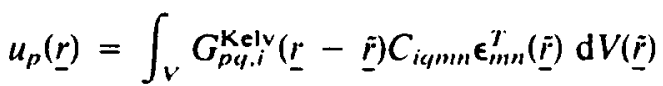

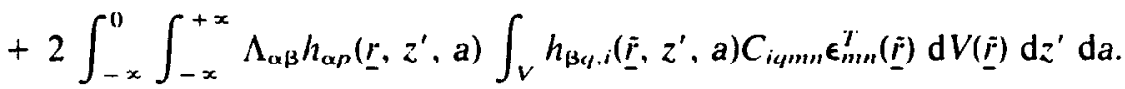

Here the $i$ in $G_{p c . i}^{\mathrm{Kelv}}$ is to be understood as differentiation on the $i$ th component of $i$. In the case of a dislocation, the expression is the same except that the integral over $V$ becomes one over $A$ and $\epsilon_{m, n}^{T} \mathrm{~d} V$ is replaced by $N_{n} \Delta u_{m} \mathrm{~d} A$.

In applications to isotropic materials, it may be noted that

$$
h_{\alpha j, l} C_{i j m n}=\mu\left(h_{\alpha m, n}+h_{\alpha r m, m}\right)+[2 v \mu /(1-2 v)] \delta_{m, n} h_{\alpha j, j} .
$$

Thus if we let the transformation strain correspond to pure dilation, $\epsilon_{m n}^{T}=\theta^{T} \delta_{m n} / 3$, then

$$
K_{\mathrm{a}}\left(z^{\prime}\right)=\frac{2 \mu(1+\nu)}{3(1-2 \nu)} \int_{\nu} h_{\alpha j_{.},}\left(\underline{r}, z^{\prime}, a\right) \theta^{T}(\underline{r}) \mathrm{d} V(\underline{r})
$$


The resulting mode I stress intensity factor is, using eqn (10),

$$
K_{1}\left(z^{\prime}\right)=\frac{2 \mu(1+\nu)}{3(1-v)} \int_{1} \frac{\partial H\left(x-a, y z-z^{\prime}\right)}{\partial y} \theta^{\prime}(x, y, z) \mathrm{d} x \mathrm{~d} y \mathrm{~d} z .
$$

where $H$ is given by eqn (8) and one may, therefore, calculate that

$$
\frac{\partial H}{\partial y}=\frac{\cos (\phi / 2)\left[1-8\left(\rho^{2} / R^{2}\right) \sin ^{2}(\phi / 2)\right]}{(2 \pi)^{3 / 2} R^{2} \rho^{1 / 2}} .
$$

Here $\rho$ is defined in eqn (17), $\tan \phi=y /(x-a)$, and

$$
R=\sqrt{(x-a)^{2}+y^{2}+\left(z-z^{\prime}\right)^{2}} .
$$

If we consider the case in which $\theta^{T}$ is independent of $z$, then integration on $z$ and some rearrangement of the result shows that

$$
K_{1}=\frac{\mu(1+\nu)}{3(1-\nu)(2 \pi)^{1 / 2}} \int_{A_{1}} \rho^{-3 / 2} \cos (3 \phi / 2) \theta^{T}(x, y) \mathrm{d} x \mathrm{~d} y
$$

where $A_{x y}$ is the cross-section of the transformed region in the $x, y$ plane. This is in agreement with the results of McMeeking and and Evans[8] and Budiansky et al..[10], derived for analysis of "transformation toughening" at crack tips. Their two-dimensional treatments correspond to a smearing-out of the actual discrete transforming particles in the $z$ direction, which may be quite adequate for the purposes addressed. The expressions (28) and (29) above allow the full three-dimensional effect of an individual transforming particle to be calculated.

As another application for the isotropic matcrial, suppose that an opening dislocation is present on the plane $y=0$ ahead of the crack. By opening dislocation is meant that only $\Delta u_{y}=\Delta u_{y}(x, z)$ is non-zero on the cut. This is surely not the most interesting crack and dislocation combination but, for it, only a mode 1 intensity factor is induced and the analysis depends only on the presently known weight function $\underline{h}_{1}$. From eqns $(25,27)$ the intensity factor distribution is

$$
K_{1}\left(z^{\prime}\right)=\int_{-\infty}^{+\infty} \int_{a}^{\infty}\left\{2 \mu h_{1, y}+[2 \nu \mu /(1-2 v)] h_{1, j,}\right\} \Delta \|_{y} \mathrm{~d} x \mathrm{~d} z
$$

and using eqn (10), this reduces to

$$
\begin{aligned}
K_{I}\left(z^{\prime}\right) & =\frac{\mu}{1-v} \int_{-\infty}^{+\infty} \int_{u}^{\infty} \frac{\partial H\left(x-a, 0, z-z^{\prime}\right)}{\partial y} \Delta u_{1}(x, y) \mathrm{d} x \mathrm{~d} z \\
& =\frac{\mu}{(2 \pi)^{3 / 2}(1-v)} \int_{-\infty}^{+\infty} \int_{a}^{\infty} \frac{\Delta u_{y}(x, z) \mathrm{d} x \mathrm{~d} z}{\sqrt{x-a}\left[(x-a)^{2}+\left(z-z^{\prime}\right)^{2}\right]} .
\end{aligned}
$$

The expression is always of one sign when $\Delta u_{y} \geqslant 0$ and shows, for example, that the opening of coplanar cracks ahead of a tensile-loaded straight-tipped main crack will always increase $K_{1}$ along the main crack tip.

Note that all the expressions for $K_{1}$ in this section are for a crack with a straight tip. They can be converted to the case of a crack with a slightly nonstraight tip by use of eqn (11).

\section{ENERGY, STRESSES, AND ENERGETIC FORCES}

Let $U$ denote the elastic strain energy of the cracked body subject to general loading by a field $\underline{f}(r)$ of body force and containing some distribution of transformation strain $\underline{\epsilon}^{T}(r) . U$ is defined by 


$$
U=\frac{1}{2} \int\left(u_{i, j}-\epsilon_{i j}^{T}\right) C_{i, m, n}\left(u_{m, n}-\epsilon_{m n}^{7}\right) \mathrm{d} V .
$$

where the integral extends over all the body. From the definition of the energy release rate $G$ of eqn (2), it is evident that

$$
\delta U=\int_{V} f_{j} \delta u_{j} \mathrm{~d} V-\int_{-\infty}^{+\infty} \Lambda_{\alpha \beta} K_{\alpha} K_{\beta} \delta a \mathrm{~d} z
$$

for crack advance $\delta a$ in presence of fixed transformation strain. Also, a simple calculation based on eqns (35), (18), (19) and (20) shows that if we alter the distribution of transformation strain at fixed crack position, then

$$
\delta U=\int_{V} f_{j} \delta u_{i} \mathrm{~d} V-\int_{V} \sigma_{i, j} \delta \epsilon_{i, j}^{T} \mathrm{~d} V
$$

Later we shall be concerned with singular $\epsilon^{T}$ corresponding to dislocations. The quantities $G\left(=\Lambda_{\alpha \beta} K_{\alpha} K_{\beta}\right)$ and $\sigma_{i j}$ can evidently be interpreted as energetic forces respectively conjugate to crack advance $\delta a$ and transformation $\delta \epsilon_{i, j}^{T}$.

If we now define the potential energy

$$
P=U-\int_{V} f_{j} u_{j} \mathrm{~d} V
$$

then one may write in general that

$$
\delta P=-\int_{V} u_{i} \delta f_{i} \mathrm{~d} V-\int_{-\infty}^{+\infty} \Lambda_{\alpha \beta} K_{\alpha} K_{\beta} \delta a \mathrm{~d} z-\int_{V} \sigma_{i j} \delta \epsilon_{i j}^{r} \mathrm{~d} V,
$$

when any of $\underline{f}$, crack position and $\underline{\epsilon}^{T}$ are varied. If $\underline{f}$ and $\underline{\epsilon}^{T}$ are held fixed, we can integrate on a and write

$$
P=P^{0}+\int_{-\infty}^{a} \frac{\partial P}{\partial a} \mathrm{da}
$$

for the potential energy when the crack tip lies parallel to the $z$ axis at $x=a$. Here $P^{v}$ denotes the potential energy which $\underline{f}$ and $\underline{\epsilon}^{T}$ cause in the uncracked body; $P^{0}$ may be formally unbounded especially if point forces, Volterra dislocations (i.e. discontinuous change of $\Delta u$ to zero at edge of $A$ ), and the like are considered. However, $\partial P / \partial a$ is bounded unless the crack plane happens to pass through a singularity such as a point of force application or a segment of dislocation line. Clearly, the middle term on the right in eqn (39) is $(\partial P / \partial a) \delta a$, and thus

$$
P=P^{0}-\int_{-\infty}^{a} \int_{-\infty}^{+\infty} \Lambda_{\alpha \beta} K_{\alpha}(z, a) K_{\beta}(z, a) \mathrm{d} z \mathrm{~d} a,
$$

where

$$
K_{\alpha}(z, a)=\int_{V}\left[h_{\alpha j}(\underline{r}, z, a) f_{j}(\underline{r})+h_{\alpha i, j}(\underline{r}, z, a) C_{i j m n} \epsilon_{m n}^{T}(\underline{r})\right] \mathrm{d} V(\underline{r})
$$

is the $\alpha$ intensity factor induced at $z$ along the crack front, when the front is at $x=a$, by $\underline{f}$ and $\underline{\epsilon}^{T}$. The crack necessarily lowers the potential energy from that for the uncracked body and the energy change is given by the integral term in eqn (41).

Since $-u_{j}(r)$ and $-\sigma_{m, m}(r)$ are the differential coefficients of $\delta P$ with respect to variations $\delta f_{, i}(r)$ and $\delta \epsilon_{m, n}^{r}(\underline{r})$, it follows from $(41,42)$ that 


$$
\begin{gathered}
u,(\underline{r})=u_{j}^{0}(\underline{r})+2 \int_{-\infty}^{u} \int_{-\infty}^{+\infty} \Lambda_{\alpha \times \beta} h_{\alpha, j}\left(\underline{r}, z^{\prime}, a\right) K_{\beta}\left(z^{\prime}, a\right) \mathrm{d} z^{\prime} \mathrm{d} a . \\
\sigma_{m m}(\underline{r})=\sigma_{m m}^{0}(\underline{r})+2 \int_{-\infty}^{a} \int_{-\infty}^{+\infty} \Lambda_{\alpha \beta} h_{\alpha i, j}\left(\underline{r}, z^{\prime}, a\right) C_{i j m m} K_{\beta}\left(z^{\prime}, a\right) \mathrm{d} z^{\prime} \mathrm{d} a .
\end{gathered}
$$

Here again, the superscript " 0 " denotes the field in the uncracked body subjected to the given distribution of $\underline{f}$ and $\underline{\epsilon}^{I}$.

Now, recognizing that the $\bar{K}^{\prime}$ s depend on $f$ and $\epsilon^{T}$. as in eqn (42), eqn (43) above for $u$ is seen to be consistent with what was given earlier in eqns (14) and (26). Those equations followed from the second property enunciated at the outset for weight functions, and given as eqn (6). However, the development of this section has made use only of the first property, eqn (5), and of relations for elastic energy. Hence the developments in this section show that the second property, eqn (6), follows from the first. As is to be expected, the expression for $\sigma_{m n}$ is consistent with multiplying $u_{t .}$ with $C_{m n i j}$ (and using $C_{m n i j}=C_{i j m m}$ ); the part $C_{m m i j} \epsilon_{i,}^{T}$ which has to be subtracted off is already included in $\sigma_{m, n}^{0}$ for the uncracked body, i.e.

$$
\sigma_{m n}^{0}=C_{m m i j}\left(u_{j, i}^{0}-\epsilon_{i j}^{T}\right)
$$

When the transformation strain corresponds to a dislocation with singular $\epsilon^{\prime}$, as in eqn $(24)$, the last term in eqns $(37,39)$ reduces to $\int_{A} N_{i} \sigma_{i j} \delta\left(\Delta u_{j}\right) \mathrm{d} A$ so that $N_{i} \sigma_{i}$ on $A$ is the energetic force conjugate to dislocation $\Delta u_{j}$. For dislocations in crystals, one is most often concerned with the Volterra case $\Delta \underline{u}=$ constant on $A$, and the alteration of the "transformation" consists of enlarging the dislocated surface $A$. When this enlargement is confined to a plane we may describe it by saying that the perimeter $\operatorname{arc} L$ of $A$ is advanced by some distance $\delta n$ normal to itself, where $\delta n$ varies with position along $L$. We then may write the last term in eqns $(37,39)$ as $\int_{L} q \delta n \mathrm{~d} L$ where $q$ is the energetic force on the dislocation and is given formally by

$$
q=N_{m} \sigma_{m n} \Delta u_{n}
$$

(this is the component of the Peach-Koehler force in a direction locally perpendicular to $L$ and $N$ ). As is well known, this formula for $q$ is meaningless as it stands for Volterra dislocations, as is also the integral over $A$ above, because of the singularity in $\sigma_{\ldots, n}$ along the dislocation edge. However, the singularity resides entirely in the term $\sigma_{m, n}^{()}$ for the uncracked body and if we write $\sigma_{m n}=\sigma_{m n}^{i}+\sigma_{m n}^{i}$, where $\sigma_{m n}^{i}$ is given by the integral term in eqn (44), then $\sigma_{m i n}^{\prime \prime}$ is finite everywhere except along the crack front. Similarly, we may write the force on a dislocation line as $q=q^{0}+q^{c}$. The first term, $q^{0}$ is well defined only in the context of some core "cut-off" procedure (at least such is required at all points of $L$ having non-zero curvature), whereas $q^{\prime}$ which represents the effect of the crack on the energetic force is independent of such procedures and is given by

$$
q^{c}=N_{m} \sigma_{m m}^{c} \Delta u_{n}
$$

Such ideas as outlined should enable the more rigorous incorporation of three-dimensional dislocation effects in the analysis of shear dislocation nucleation from a crack tip[11]. They should also provide a fuller understanding of the dislocation-crack tip interaction, thus far studied two-dimensionally[12]. These problems can, however, be addressed in the requisite generality only when all three weight functions $\underline{h}_{\alpha}$ are employed.

As a simpler illustration, the stress $\sigma_{y y}$ on $y=0$ is now calculated for an arbitrary distribution of opening dislocation $\Delta u_{y}(x, z)$ on $y=0$ ahead of the tip (which is taken to be at $x=a=0$, i.e. coincident with the $z$ axis). The stress intensity due to this opening is given by eqn (34). Only mode 1 is involved and we denote the intensity factor as $K_{1}\left(z^{\prime}, a\right)$. Then using eqns (3) and (27) for the isotropic material, eqn (44) for $\sigma_{m n}$ gives 


$$
\begin{aligned}
\sigma_{m n}(\underline{r})=\sigma_{m, n}^{0}(\underline{r})+(1-v) \int_{-x}^{0} \int_{-x}^{+x}\left\{h_{1 m . n}\left(\underline{r}, z^{\prime}, a\right)+h_{1 n, m}\left(\underline{r}, z^{\prime}, a\right)\right. \\
\left.+[2 \nu /(1-2 \nu)] \delta_{m, m} h_{1 j, j}\left(r, z^{\prime}, a\right)\right\} K_{1}\left(z^{\prime}, a\right) \mathrm{d} z^{\prime} \mathrm{d} a .
\end{aligned}
$$

Thus, by eqn (10), the tensile stress on $y=0$ is

$$
\sigma_{y y}(x, 0, z)=\sigma_{y y}^{0}(x, 0, z)+\int_{-\infty}^{0} \int_{-x}^{+x} \frac{\partial H\left(x-a, 0, z-z^{\prime}\right)}{\partial y} K_{1}\left(z^{\prime}, a\right) \mathrm{d} z^{\prime} \mathrm{d} a,
$$

and by using eqn (30) for $\partial H / \partial y$ and eqn (34) for $K_{1}$ this becomes

$$
\begin{aligned}
\sigma_{y y}(x, 0, z)= & \sigma_{y y}^{0}(x, 0, z) \\
& +\frac{\mu}{(2 \pi)^{3}(1-v)} \int_{-x}^{0} \int_{-x}^{+x} \int_{-x}^{+\infty} \int_{0}^{x} \frac{\Delta u_{y}(\bar{x}, \bar{z}) \mathrm{d} \tilde{x} \mathrm{~d} z \mathrm{~d} z^{\prime} \mathrm{d} a}{\rho^{1 / 2} \dot{\rho}^{1 / 2} R^{2} \dot{R}^{2}}
\end{aligned}
$$

for $x>0$, where

$$
\rho=x-a, \dot{\rho}=\bar{x}-a, R^{2}=(x-a)^{2}+\left(z-z^{\prime}\right)^{2}, \dot{R}^{2}=(\tilde{x}-a)^{2}+\left(\tilde{z}-z^{\prime}\right)^{2} .
$$

The integral on $z^{\prime}$ can be done with the help of eqn (16), resulting in

$$
\frac{\mu}{8 \pi^{2}(1-\nu)} \int_{-x}^{0} \int_{-x}^{+x} \int_{0}^{x} \frac{(x+\bar{x}-2 a) \Delta u_{y}(\tilde{x}, \tilde{z}) \mathrm{d} \tilde{x} \mathrm{~d} \tilde{z} \mathrm{~d} a}{(x-a)^{3 / 2}(\bar{x}-a)^{3 / 2}\left[(z-\bar{z})^{2}+(x+\bar{x}-2 a)^{2}\right]}
$$

for the last term in eqn (50) above. The integral on a is reduced to an elementary form by introducting a new variable

$$
t=2(x-a)^{1 / 2}(\tilde{x}-a)^{1 / 2},
$$

which runs from $x$ to $2(x \bar{x})^{1 / 2}$ as a runs from $-x$ to zero. Thus the integral term is

$$
\frac{\mu}{2 \pi^{2}(1-v)} \int_{-\infty}^{+\infty} \int_{0}^{x} \int_{2(x, x) 1 / 2}^{\infty} \frac{\Delta u_{y}(\tilde{x}, \bar{z}) \mathrm{d} t \mathrm{~d} \bar{x} \mathrm{~d} \bar{z}}{t^{2}\left[t^{2}+(x-\bar{x})^{2}+(z-\bar{z})^{2}\right]},
$$

which is readily evaluated with the substitution $t=D / \tan \phi$ where

$$
D=\left[(x-\tilde{x})^{2}+(z-\tilde{z})^{2}\right]^{1 / 2} .
$$

Thus the final expression for stress is

$$
\begin{aligned}
\sigma_{y y}(x, 0, z)= & \sigma_{y y}^{0}(x, 0, z) \\
& +\frac{\mu}{2 \pi^{2}(1-\nu)} \int_{-x}^{+\infty} \int_{0}^{x} \frac{1}{D^{3}}\left[\frac{D}{2(x \bar{x})^{1 / 2}}-\arctan \frac{D}{2(x \bar{x})^{1 / 2}}\right] \\
& \times \Delta u_{y}(\bar{x}, \bar{z}) \mathrm{d} \bar{x} \mathrm{~d} \bar{z} .
\end{aligned}
$$

Note that the integrand remains finite as $\bar{x}, \bar{z}$ approach the point $x, z$ at which $D=0$.

The expression $\sigma_{y y}^{0}$ for the stress which the same opening $\Delta u_{y}$ induces in an infinite uncracked body can be found by several routes. Perhaps it is simplest to follow Meade and Keer[7] and their predecessors in observing that such elasticity problems are governed by a three-dimensional harmonic function $Y=Y(x, y, z)$ having the properties that on the plane $y=0$

$$
\sigma_{y}^{\prime \prime}(x, 0, z)=-\partial Y /\left.\partial y\right|_{y=0}, \quad \Delta u_{y}(x, z)=-\left.|2(1-\nu) / \mu| Y\right|_{y=0+} .
$$


Thus we seek a harmonic function $Y$ which vanishes at infinity and takes on given values on the plane $y=0$. Some elementary analysis shows that the solution can be obtained by differentiating the spherically symmetric harmonic potential $\left(D^{2}+y^{2}\right)^{-1 / 2}$ on $y$ (i.e. forming a double layer) and superposing such that

$$
Y(x, y, z)=\frac{\mu}{4 \pi(1-v)} \frac{\partial}{\partial y} \int_{-\infty}^{+\infty} \int_{0}^{x} \frac{\Delta u_{y}(\tilde{x}, \tilde{z}) \mathrm{d} \tilde{x} \mathrm{~d} \tilde{z}}{\left(D^{2}+y^{2}\right)^{1 / 2}}
$$

and hence

$$
\begin{aligned}
\sigma_{y y}^{0}(x, 0, z) & =-\frac{\mu}{4 \pi(1-\nu)}\left[\frac{\partial^{2}}{\partial y^{2}} \int_{-\infty}^{+\infty} \int_{0}^{\infty} \frac{\Delta u_{y}(\tilde{x}, \tilde{z}) \mathrm{d} \tilde{x} \mathrm{~d} \bar{z}}{\left(D^{2}+y^{2}\right)^{1 / 2}}\right]_{y=0} \\
& =\frac{\mu}{4 \pi(1-\nu)}\left(\frac{\partial^{2}}{\partial x^{2}}+\frac{\partial^{2}}{\partial z^{2}}\right) \int_{-\infty}^{+\infty} \int_{0}^{\infty} \frac{\Delta u_{y}(\tilde{x}, \tilde{z})}{D} \mathrm{~d} \bar{x} \mathrm{~d} \bar{z}
\end{aligned}
$$

where $D$ is given by eqn (53). By moving one $\partial / \partial x$ in eqn (57) inside the integral, observing that

$$
\frac{\partial}{\partial x}\left(\frac{1}{D}\right) \Delta u_{y}=-\frac{\partial}{\partial \bar{x}}\left(\frac{1}{D}\right) \Delta u_{y}=-\frac{\partial}{\partial \dot{x}}\left(\frac{\Delta u_{y}}{D}\right)+\frac{1}{D} \frac{\partial \Delta u_{v}}{\partial \dot{x}} .
$$

applying the divergence theorem while noting that $\Delta u_{y}$ vanishes outside some finite region, doing the same for one $\partial / \partial z$, and then moving the other $\partial / \partial x$ and $\partial / \partial z$ inside the integral one has

$$
\sigma_{y y}^{0}(x, 0, z)=\frac{\mu}{4 \pi(1-\nu)} \int_{-\infty}^{+\infty} \int_{0}^{x}\left[(\tilde{x}-x) \frac{\partial}{\partial \dot{x}}+(\tilde{z}-z) \frac{\partial}{\partial \dot{z}}\right] \Delta u_{y}(\tilde{x}, \tilde{z}) \frac{\mathrm{d} \tilde{x} \mathrm{~d} \tilde{z}}{D^{3}},
$$

which is the form given by Weaver[13].

Equations (54) with (57) or (59) gives the stress induced in the cracked solid by some arbitrary distribution of opening dislocation on $y=0$. One must add to that expression the stress field induced in the cracked solid by whatever loadings $f$ act in order to obtain the total stress. If, as an example, one wishes to analyze the opening $\Delta u_{y}$ of a crack on some region $A$ of the half plane $y=0, x>0$, ahead of the main crack on $y=0, x<0$, then it is necessary that $\Delta u_{y}$ be chosen so that the total $\sigma_{y y}$ vanishes on $A$. Weaver[13] has outlined a discretization of the integral in eqn (59) which allows numerical solution for the opening $\Delta u_{y}$ of an isolated crack on $A$ in an infinite but otherwise uncracked body. By adding to Weaver's discretized matrix the nonsingular contributions from the integral in eqn (54) on $A$ it would seem straightforward to extend his approach to determine numerically the opening $\Delta u_{y}$ of the crack surface $A$ ahead of the half-plane crack.

Acknowledgement-This study was supported by the NSF Materials Research Laboratory at Harvard University.

\section{REFERENCES}

1. J. R. Rice, First order variation in elastic fields due to variation in location of a planar crack front. $J$. Appl. Mech., 52, 3 (1985), in press (ASME preprint 85-APM-25).

2. H. F. Bueckner, A novel principle for the calculation of stress intensity factors, $Z$. angen'. Math. Mec $h$. 50, $529-533(1970)$

3. H. F. Bueckner, Field simgularities and related integral representations. In Mechanics of Frachure I: Methods of Analysis and Solution of Crack Problems (Edited by G. C. Sih), pp. 239-314. Noordhoff. Leyden (1972).

4. J. R. Rice, Some remarks on elastic crack tip stress fields, Int. J. Solids Structures 8, 751-758 (1972).

5. A. N. Stroh, Dislocations and cracks in anisotropic elasticity. Phil. Mag. 3, 625-646 (1958). 
6. D. M. Barnett and R. J. Asaro, The fracture mechanics of slit cracks in anisotropic elastic media. $J$. Mech. Phys. Solids 20, 353-366 (1972).

7. K. P. Meide and L. M. Keer. Stress intensily factors for a semi-infinite plane crack with a wavy front. J. Elasticity 14, 79-92 (1984).

8. R. M. McMecking and A. G. Evans. Mechanics of transformation toughening in brittle materials. $J$. Amer. Ceram. Soc'. 65, 242-246 (1982).

9. J. W. Hutchinson. On steady quasi-static crack growth. Harvard University, Division of Applied Sciences. Report DEAP S-8 (April 1974).

10. B. Budiansky. J. W. Hutchinson. and J. C. Lambropoulos. Continuum theory of dilatant transformation loughening in ceramics, Imt, J. Solid.s Structures 19, 337-355 (1983).

11. J. R. Rice and R. Thomson. Ductile versus brittle behavior of crystals, Phil. Mag. 29, 73-97 (1974)

12. J. R. Rice. Conserved integrals and energetic forces. In Fundamentals of Deformation and Fracrure $(J$. D. Eshe!hy Memorial Symposium) (Edited by B. A. Bilby. K. J. Miller, and J. R. Willis) pp. 33-56. Cambridge University Press. (1985).

13. J. Weaver. Three-dimensional crack analysis. Int. J. Solids Structures 13, 321-330 (1977)

\section{Note added in proof:}

Since preparation of the manuscript the author has learned that $\mathrm{Dr}$. H. F. Bueckner has derived, in the paper The weight functions of mode $I$ of the penny-shaped and of the elliptic crack (Fracture Mechanics and Technology, (Edited by Sih and Chow), Vol. 2, pp. 1069-1107, Sijthoff and Noordhoff, (1977)), results somewhat analogous to those quoted here from [1] as eqns (7)-(9) for the mode I weight function of the halfplane crack. Bueckner's results are for fields which vary as $\cos (\lambda z)$ along the crack front, but by weighting them with the Fourier transform of a Dirac function of $z-z^{\prime}$ and then integrating in $\lambda$ it is possible to derive the mode 1 weight function of eqns (7)-(9) from them. As learned in a private communication from $\mathrm{Dr}$ Bueckner, he has independently derived the mode 1 weight function in this way, in as yet unpublished work, and has developed a similar derivation for the mode 2 and mode 3 weight functions denoted $h_{2}$ and $h_{3}$ here and given only by integral representations in [1]. His results update the discussion at the bottom of the second page of this paper. 